\title{
Die zweite Welle
}

\section{Oft sind es die Krisen, die Veränderungen erzwingen. Die aktuelle Pandemie bringt jedenfalls die Digitale Transformation in Deutschland voran.}

Die zweite Welle, sie ist da! - Nein, ich meine nicht die zweite Welle der COVID-19-Erkrankungen. Ich meine eine zweite Welle der Digitalen Transformation, die in diesem Jahr in breiten Kreisen der Bevölkerung angekommen ist, als Folge der Abwehrmaßnahmen gegen die Ausbreitung der Infektion. Nur, es ist in meinen Augen wirklich bedauerlich, dass dazu eine solche Krise notwendig war.

Videokonferenzen etwa sind im professionellen Bereich seit vielen Jahren an der Tagesordnung. Sicher, es gibt da immer wieder Pleiten und Pannen, das kann einen zur Weißglut treiben, wenn man wichtige Geschäftsthemen besprechen will. Die einschlägigen YouTube-Videos, z.B. A Video Conference in Real Life, sind da wirklich treffend. Aber ohne diese digitale Technik hätte ich mein Berufsleben über viele Jahre im Flieger verbringen müssen. Und das finde ich wirklich nicht erstrebenswert - nein, ich brauche keine HON-Karte bei Miles\&More für mein Ego.

Die Digitalisierung von Geschäftsprozessen ist eine weitere Entwicklung, die aus der Wirtschaft nicht mehr wegzudenken ist. Personalverwaltung, Finanzen, Einkauf, Logistik, all das ist doch digitalisiert und hoffentlich auch integriert. Nur so können wir schnell genug agieren, um im globalen Wettbewerb mitzuspielen.

Im Alltagsleben allerdings ist vieles davon noch nicht angekommen, und das besonders in Deutschland. Nicht nur Drogenhändler und betrügerische Banker bezahlen ihre Rechnungen am liebsten bar: Cash und keine Fragen. In Skandinavien dagegen kauft man auch sein Eis bargeldlos. Nicht falsch verstehen: Bargeld ist eine großartige Sache und sollte nicht abgeschafft werden. Aber für das Eiscafé oder den Discounter sind doch digitale Zahlungsmittel viel praktischer, da mag ich nicht mit Münzen hantieren.

E-Learning-Programme sind bereits weit verbreitet und zum Teil auch hervorragend gemacht. Nur unsere Schulen sind auf so etwas in keiner Weise eingestellt und müssen improvisieren.

Und dann gibt es da unser deutsches Gesundheitswesen, auf das wir zu Recht alle stolz sind. Seltsam aber: Da vertrauen erstaunlich viele Menschen eher den kruden Ideen des Samuel Hahnemann als der Digitalen Gesundheitskarte. Laborbefunde werden aus Gründen der „Datensicherheit“" per Brief oder Fax übermittelt, um dann beim Empfänger womöglich wieder eingetippt zu werden, hoffentlich fehlerfrei. Tradition und vermeintlich Bewährtes werden hochgehalten, auch wenn das jeglichen wissenschaftlichen Erkenntnissen zuwiderläuft. Und die Verschwörungstheoretiker küren sich mit Bill Gates ausgerechnet einen der Protagonisten der Digitalisierung zum Erzfeind. Warum nur?

Immerhin, wir haben die CORONA-Warn-App, um die uns sogar Boris Johnson beneidet. Die Zahl der Downloads ist beachtlich, aber dennoch weit von den $60 \%$ entfernt, die eigentlich erforderlich wären, um einen durchschlagenden Effekt zu erzielen. Auch hier viel Misstrauen gegenüber der digitalen Technik, obwohl es ja eigentlich nicht die Technik ist, die uns bedroht, sondern die Menschen, die sie missbrauchen könnten. Immerhin ein weiteres Beispiel dafür, wie uns die Digitale Transformation hilft, die Krise zu meistern.

Wie steht es also mit unserer Digitalkompetenz? Schlecht, denke ich, wenn in Filmen und Medien immer noch der abgedrehte Nerd gezeigt wird, der im finsteren Keller in weltweite Datensysteme eindringt, während die wirklichen Akteure stolz beteuern, von Mathe und Technik keine Ahnung zu haben. Es macht mir Sorge, wenn Zeitungen uns digitale Entgiftung empfehlen. Digitalisierung sei böse und bedrohlich, das ist leider oft der Tenor.

Aber wir sollten nicht verzagen. Es gibt ja Digitalen Nachwuchs oder Talents4Future, wie es die LMU genannt hat. Unter diesem Namen firmiert eine Plattform, auf der Studierende ihre digitalen (und analogen) Kompetenzen sortieren können. Sie können sehen, welche digitalen Technologien und Geschäftsmodelle gerade gefragt sind, sie können ein Profil anlegen und erfahren dabei mehr über ihre Persönlichkeit. Und die Wirtschaft kann auf diesem Portal tatsächlich Digitalen Nachwuchs finden und ihren Bedarf darstellen. Dass Talente und Bedarf zusammenkommen, dafür sorgt hier Künstliche Intelligenz - das ist Stand der Digitaltechnik.

Die Digitale Transformation ist jedenfalls nicht aufzuhalten.

\section{Dr. Lothar Borrmann}

\section{Dr. Lothar Borrmann}

Dr. Lothar Borrmann war bis 2019 in der Siemens AG weltweit für Innovation im Bereich Software- und Systemtechnik verantwortlich. Er unterstützt derzeit die LMU als unabhängiger Berater bei der Nutzung digitaler Technologien.

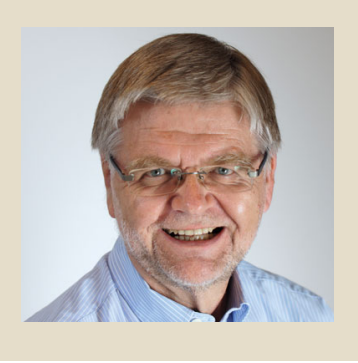

\section{Die DIGITALE WELT erscheint ab der nächsten Ausgabe ausschließlich digital. Sichern Sie sich JETZT Ihr kostenloses Abo unter digitaleweltmagazin.de/magazin/}

